\begin{tabular}{|c|c|c|c|c|c|c|c|c|c|c|c|c|c|c|c|c|c|c|c|c|c|}
\hline \multicolumn{2}{|c|}{ I 9 II } & \multicolumn{3}{|c|}{ t. m. Teramo } & \multicolumn{2}{|c|}{$\Lambda \alpha$} & \multicolumn{3}{|c|}{$\alpha \delta$} & f. & \multicolumn{3}{|c|}{$\alpha$ app. } & $\log p \cdot A$ & \multicolumn{3}{|c|}{$\delta$ app. } & $|\log p \cdot d|$ & \multicolumn{2}{|c|}{ Red. ad l. app. } & * \\
\hline & & & & & -0 & $5^{5} \times 4$ & $T$ & & 46.8 & 5,10 & $2 I^{h}$ & & & $9 \cdot 397 n$ & $+34^{\circ}$ & $28^{\prime}$ & I 1.8 & $22 \mathrm{I}$ & +25.39 & + & \\
\hline & 16 & 9 & 30 & 47 & $+I$ & 36.14 & - & & 24.5 & $9, \mathrm{I} I$ & $2 \mathrm{I}$ & 14 & & $9.455 \mathrm{n}$ & $+4 I$ & 10 & 17.4 & & +2.4 .3 & +7.7 & \\
\hline & 20 & 8 & 54 & I 5 & +0 & 57 & - & & & I I & 2 I & 5 & & & +42 & 55 & & & .42 & +8.2 & \\
\hline & 3 & 9 & I I & 42 & $-\mathbf{I}$ & 40. & - & I 5 & $5 \mathrm{I.I}$ & & 20 & 49 & & & +45 & & & & .39 & +9.1 & \\
\hline & 5 & I 2 & 22 & 50 & -0 & I & + & 95 & & $* 20,6$ & 20 & 35 & 48 & & +47 & 32 & .0 & & 34 & +9.7 & \\
\hline & 27 & 8 & 19 & 24 & -0 & & - & 25 & & $* 8,8$ & 20 & & & & +49 & 8 & & $I_{n}$ & 28 & +10.3 & \\
\hline & 29 & $\mathrm{I}^{2}$ & I 3 & I 4 & -0 & IC & - & 21 & 10.0 & $* 8,8$ & 20 & 6 & 0 & & +50 & 57 & 2 & $5 n$ & 8 & .8 & \\
\hline \multirow[t]{8}{*}{ Sett. } & I & 8 & 3 & 22 & -0 & 22.98 & + & 04 & 44.0 & $* 9,9$ & 19 & 40 & 37 & & +53 & 7 & 8 & $5 n$ & o & .6 & \\
\hline & 4 & I 3 & 45 & $2 \mathrm{I}$ & +0 & 15 & + & 03 & 3 & $* 9, \mathrm{r} \circ$ & 19 & 6 & & 9.8 & +55 & I I & & & 3 & & \\
\hline & 6 & I I & 8 & 27 & +0 & 32.09 & + & 7 & I9. & 30,8 & 18 & 44 & 95 & & +56 & 7 & 5 & & 2 & & \\
\hline & 8 & I I & $5^{8}$ & & +0 & 29.39 & +1 & & 33. & 24,6 & 18 & 19 & 4 & & +56 & 50 & 49.4 & & 8 & +1 & \\
\hline & 10 & 9 & 2 & 54 & +3 & 9.10 & + & 83 & 34. & 23,8 & 17 & 55 & & & +57 & I 2 & $5^{8.5}$ & $86_{n}$ & 3 & + & \\
\hline & 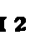 & I 2 & 20 & 6 & +0 & $6.2 \mathrm{I}$ & + & 4 & & $* 6,8$ & 17 & 27 & & & +57 & I 5 & & & +0.80 & +10.5 & \\
\hline & & 8 & I 2 & 35 & -2 & 23.60 & - & I : & $5^{8.2}$ & 25,5 & I 5 & 48 & & & +54 & 6 & 28.9 & & +0.19 & +5.3 & \\
\hline & 9 & 7 & 32 & 41 & $+I$ & 35.42 & + & 5 & 39.2 & 35,7 & 14 & 22 & 53.62 & 9.789 & +45 & $3^{8}$ & $35 \cdot \mathrm{I}$ & 0.572 & +o.1 7 & $-\quad 1.7$ & \\
\hline
\end{tabular}

Cometa 19 I I (Quenisset).

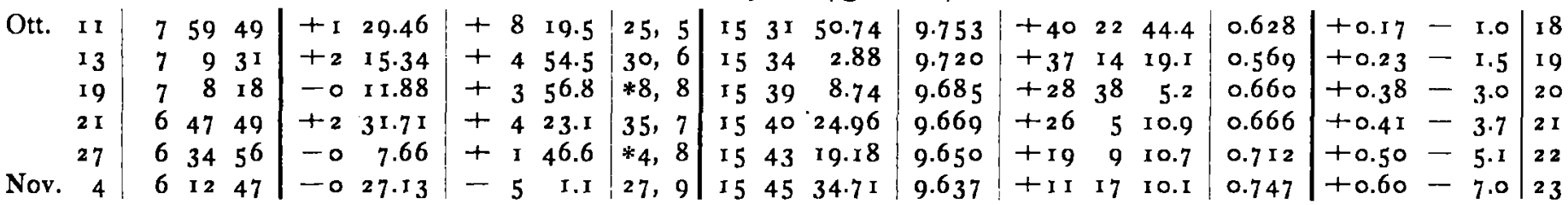

Gli asterischi * nella quinta colonna significano che i corrispondenti $\boldsymbol{A} \alpha$ furono misurati, come i $\boldsymbol{A} \delta$, mediante la vite micrometrica.

Posizioni medie delle stelle di confronto.

\begin{tabular}{|c|c|c|c|}
\hline * & $\alpha 1911.0$ & $\delta$ I 911.0 & Autorità \\
\hline I & $22^{\text {h }} \quad 3^{\mathrm{m}} 57^{\text {s.o }} \cdot 9$ & $+26^{\circ} 34^{\prime} 40^{\prime \prime} 6$ & AG Cbr E. I $3^{2}$ I I \\
\hline 2 & 2 I 4544.30 & $+3^{2} 5348.6$ & AG Lei 9 r 45 \\
\hline 3 & 214158.18 & +3422 19.2 & 9116 \\
\hline 4 & $21 \quad 1243.55$ & +4 I 534.2 & $A G$ Bo I 5276 \\
\hline 5 & 2 I $4 \quad 4 \quad 5.9$ I & $+425^{8} 44.9$ & I 5095 \\
\hline 6 & $20 \quad 50 \quad 45.54$ & +453955.8 & I 48 I6 \\
\hline 7 & 2036 & +472222.3 & I 4504 \\
\hline 8 & $20 \quad 2314.62$ & +49 I I I 3.3 & 14213 \\
\hline 9 & $\begin{array}{lll}20 & 6 & 9.3^{8}\end{array}$ & +505916.4 & $\mathrm{AG} \mathrm{Cbr} \mathrm{M.} 6382$ \\
\hline IO & $19 \quad 40 \quad 58.28$ & $+5365^{1.2}$ & 6 I 64 \\
\hline I I & $\begin{array}{lll}19 & 6 & 30.67\end{array}$ & +55 I I 10.1 & AG Hels 102 I $5 \mathrm{dpl}$. \\
\hline I 2 & I $844 \quad$ I 7.34 & 022.2 & 9974 \\
\hline
\end{tabular}

Teramo, Osservatorio Collurania, 5 Novembre 191 I.

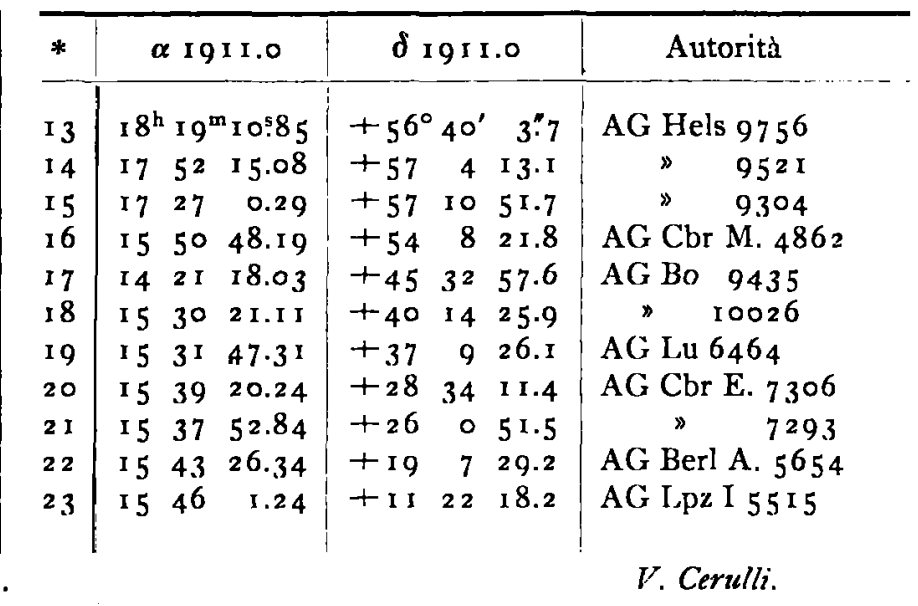

\title{
Mitteilungen über Veränderliche.
}

Bemerkung $z u$ 6.1907 Andromedae.

Im Harvard-Zirk. 124 (A. N. 4 I 59) wird für den Stern als Amplitude seines Lichtwechsels das Intervall $8^{\mathrm{m}}$. o bis $<\mathrm{I} 3^{\mathrm{m}}$ phot. angegeben. Ich habe den Stern seit der Ent. deckungsanzeige sporadisch beobachtet aber nie gesehen. Bei der letzten Beobachtung ( I 9 I I Nov. I 9 ) war der Stern sicher < $\mathbf{2}^{\mathrm{m}}$, bei der folgenden ( 19 I I Dez. 22) stand nördlich von der in der Entdeckungsanzeige angegebenen Stelle ein gelbroter Stern etwa $9 \div 5$. Die Helligkeit desselben wurde bis zum 28. Januar 1912 öfters bestimmt und als konstant gefunden. Die photographische Helligkeit ist bedeutend schwächer, so daß die obere Grenze der Entdeckungsanzeige für visuelle Beobachtungen höher zu legen ist. Falls dieser Stern mit dem Harvard-Stern identisch ist, so ist sein Verhalten höchst interessant. Der Stern würde in vier Wochen drei Größenklassen zugenommen haben, um dann durch die lange Zeit konstant zu bleiben, was sehr auffallend ist. Bei der hypothetischen Lichtkurve des Jahres rg I I-r2 müßte der Lichtwechsel wahrscheinlich irregulär sein und außerdem müßte auch die Helligkeit der maximalen Phase äußerst stark von einer Erscheinung zur anderen schwanken. Da bei dem ungünstigen Klima unseres Observatoriums eine längere Pause 
in der Beobachtungsreihe eintreten könnte, bittet der Unterzeichnete, diesem Sterne Aufmerksamkeit widmen zu wollen.

Observatorium Nižbor, rgra Jan. $29 . \quad$ L. Pracka.

Neuer Veränderlicher $\mathrm{BD}+6^{\circ} 975=2.1912$ Orionis. $5^{\mathrm{h}} 30^{\mathrm{m}} 9.43+6^{\circ} 13^{\prime}$ I $3^{\prime \prime}$. $\quad$ ( 1855.0$)$

$53234.20+6$ I 5 5.6 (1900.0) AG Lpz II 2300

Beim Suchen nach dem Planeten 5 I Nemausa vermißte ich 19 г Dez. I 8 den Stern BD + $6^{\circ} 9759^{\mathrm{m}}$.o, während sein Nachbar $B D+6^{\circ} 96^{\circ} 8^{\mathrm{m}} \cdot 9$ und auch $B D+6^{\circ} 9799^{m} \cdot 5$ sichtbar waren. Bei besserem Luftzustande erschien I9 2 Jan. I 7 der Stern $+6^{\circ} 975 \mathrm{I}^{1 / 2}$ Größenklassen schwächer als $+6^{\circ} 976$. In der Skala der BD ergab sich durch Vergleichung mit $+6^{\circ} 968,976,977,979$ und 980 die Größe von $+6^{\circ} 975$ zur Zeit 1912 Jan. I 7.3 zu I $0^{m} .4$. Nach meiner unmittelbaren Größenschätzung war er sogar nur 10.8 bei nicht sehr durchsichtiger Winterluft. Die Größenschätzungen Bonn 9\%. 1887 Febr. 3 und 5 Leipzig 9 m. und 19 I 2 Jan. I 7 Düsseldorf $\mathrm{I}^{\mathrm{m}} \cdot 4$ scheinen jeden $Z$ weifel an der Veränderlichkeit dieses Sterns auszuschließen.

Düsseldorf, 1912 Jan. 18.

Wilhelm Luther.

Der Stern $B D+6{ }^{\circ} 975$ ist beobachtet von

Schmidt in der "Orion-Zone" 1851 Jan. 8

$$
9^{\mathrm{m}} \cdot 5 \quad 5^{\mathrm{h}} 30^{\mathrm{mm}} 7^{\mathrm{s}}+6^{\circ} 13 \cdot 9
$$

Krueger in der "Sucher-Zone" Nr. 3 I 8 I 854 Jan. 22 $8^{\mathrm{m}} \cdot 55^{\mathrm{h}} 3^{\mathrm{m}} 8: 8+6^{\mathrm{s}}{ }^{\mathrm{s}} 3^{\prime} \cdot 9$.

Bonn, I 912 Jan. 30.
F. Küstner.

\section{Mitteilungen über kleine Planeten.}

Folgende Planeten sind I 9 I 2 Jan. 29 von Dr. F. Rheden photographiert und am nächsten Tage von mir am Refraktor beobachtet :

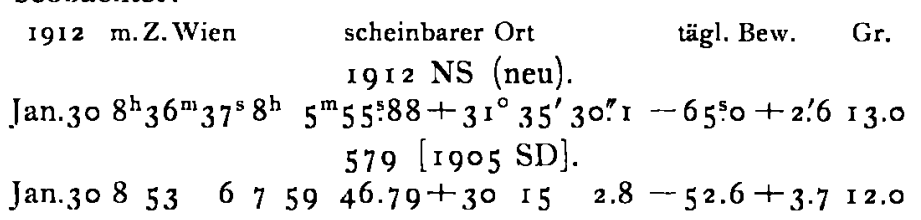

Wien, k. k. Sternwarte, r 9 I 2 Jan. 3 I.

7. Palisa.

Nach einer Mitteilung des $k$. Recheninstituts lauten die Beobachtungen von 2 I I Isolda $=$ IgI $2 \mathrm{NQ}$ nach den Klischees der Herren Fules Baillaud und Demetresco:

$$
\begin{aligned}
& 1912 \mathrm{~m} . Z \text {. Paris } \\
& \text { Jan. } 1810^{\mathrm{h}} 27^{\mathrm{m}} 6^{\mathrm{h}} 4^{\mathrm{m}} 29^{\mathrm{s}} 89+21^{\circ} 49^{\prime} 13^{\mathrm{n}} \cdot 7 \\
& \begin{array}{lllllllll}
20 & \text { I } 2 & 32 & 6 & 3 & 12.46 & +21 & 46 & 58.6
\end{array}
\end{aligned}
$$

Größe zwischen $10^{\mathrm{m}} \cdot 5$ und $\mathrm{I} \mathrm{I}^{\mathrm{m}}$. $\mathrm{o}$.

Der Planet ist von Herrn M. Giacobini am Refraktor der Pariser Sternwarte beobachtet:

$$
\begin{aligned}
& \text { IgI } 2 \quad \text { m. Z. Paris } \alpha \text { app. } \log p \cdot \Delta \quad \delta \text { app. } \quad \log p-\Delta \\
& \text { Jan. } 2410^{\mathrm{h}} 5^{2^{\mathrm{m}}} \mathrm{I}^{\mathrm{s}} 6^{\mathrm{h}} \mathrm{I}^{\mathrm{m}} 50^{\mathrm{o}} .019 .049+21^{\circ} 4^{\prime} 5^{2 !} .30 .6 \text { I I } \\
& 27 \text { ro } 57 \text { I } 6 \circ 30.149 .156+2 \text { I } 3954.7 \quad 0.624 \\
& \text { I912 } \Delta \alpha \quad \Delta 8 \quad \text { Vgl. Red.ad l. app. }
\end{aligned}
$$

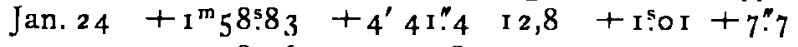

$$
\begin{aligned}
& 27+038.96+\mathrm{I} 43.8 \text { I } 2.5+\mathrm{I} .0 \mathrm{I}+7.7
\end{aligned}
$$

Größe I I m2. Vergleichstern I 9 × 2.0: $\alpha 5^{\mathrm{h}} 59^{\mathrm{m}} 50^{\mathrm{s}} \mathrm{I} 7$ $\delta+2 I^{\circ} 38^{\prime} 3^{\prime \prime} 2$ AG Berl B 2 I 6 I.

\section{Erklärung.}

Der Verlag Friedr. Vieweg \& Sohn in Braunschweig versendet eine "Vorankündigung " einer dritten Ausgabe der Theoretischen Astronomie von W. Klinkerfues, Neubearbeitung von Prof. Dr. H. Buchholz, Privatdozent an der Unjversität Halle a. S., der beigegeben ist ein Abdruck der "Vorbemerkungen zur dritten Ausgabe, mit einer kritischen Übersicht der neueren von $W$. Gibbs, $P$. Harzer und $A$. Leuschner gegebenen Methoden der Bahnrechnung «. Hierin setzt der Bearbeiter u. a. auseinander, warum er die Harzersche Methode nicht in sein Buch aufgenommen habe. Er erklärt (S. XXV), die Harzersche Methode löse zwar in rein mathe. matischer Beziehung das Problem mit viel Scharfsinn, tue das jedoch nicht in praktischer Beziehung, auf die es astronomisch ankomme, und bemerkt dazu in einer Fußnote:

"Hierzu vergleiche man die von Herrn Harzer unmittelbar vor der "Einleitung " seiner Arbeit gemachte Vorbemerkung auf S. 3, Zeile 4 und 5, wonach Winke des bekannten Mathematikers Prof. Staeckel, während dieser an der Kieler Universität wirkte, „für die Gestaltung der analytischen Darstellung der Untersuchungen nützlich gewesen"."

Karlsruhe i. B., im Januar I 9 I 2.
Hierin liegt nicht nur eine unrichtige Wiedergabe des Wortlauts der betreffenden Stelle der Harzerschen Vorbemerkung, die wörtlich so lautet:

"für die Gestaltung der analytischen Darstellung der Untersuchungen sind mir an einigen Stellen Gespräche, die ich mit meinem Kollegen, Herrn Paul Stäckel, gehabt habe, nützlich gewesen",

sondern auch eine Entstellung des Sachverhalts. Herr Harzer hat nur unserer vielen freundschaftlichen Gespräche über wissenschaftliche Gegenstände gedenken wollen, die bald dem einen, bald dem anderen Anregung gegeben haben, niemand wird aber aus seiner Äußerung den Sinn herauslesen, der bei Herrn Buchholz durch die Änderung des Wortlauts entstanden ist. Alles was die Arbeit des Herrn Harzer enthält, ist sein geistiges Eigentum; eine von mir herrührende $\mathrm{Be}$ merkung, die mit der astronomischen Untersuchung nur in losem Zusammenhang steht, ist (S. 29-30) ausdrücklich als solche bezeichnet worden. Ich verwahre mich deshalb aufs entschiedenste dagegen, daß mir von Herrn Buchholz etwas zugeschoben wird, worauf ich keinen Anspruch habe.

Inhalt zu Nr. 4554. H. Naumann. Beobachtungen von Kometen. 305. - L. Gabba. Osservazioni della Cometa 19i i b (Kiess). 3i 3. - E. Ernst. Beobachtungen des Kometen I9I I (Quénisset). $315 .-V$. Cerulli. Osservazioni delle Comete Brookss e Quénisset. 3 15. Mitteilungen ubber Veränderliche. 317. - Mitteilungen uber kleine Planeten. 319. - P. Stäckel. Erklärung. 3 I9. 